\title{
Prevalencia de embarazo ectópico en el Hospital Carlos Andrade Marín, 2017 a 2019

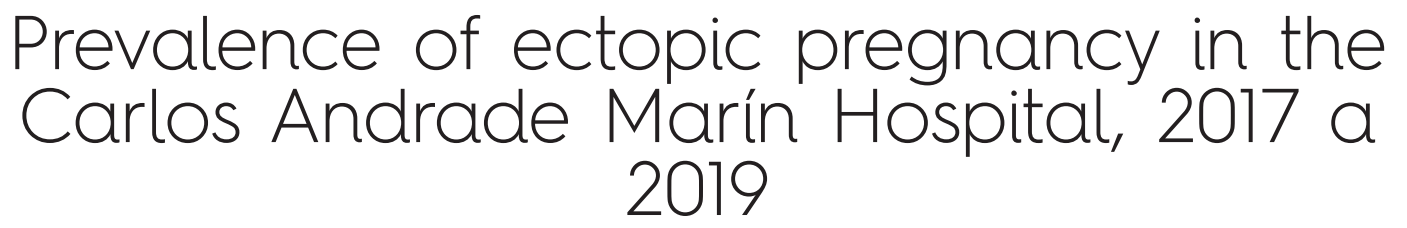

\author{
Darwin Logroño $^{1}$ iD , Jorge Ramírez ${ }^{2}$ iD , Adriana Campoverde ${ }^{3}$ \\ Ginecólogo-Obstetra; Médico Tratante en el Hospital Carlos Andrade Marín y Hospital Metropolitano; Quito, Ecuador'; \\ Médico Postgradista R4-Ginecología y Obstetricia; Hospital Carlos Andrade Marín; Quito, Ecuador²: \\ Médico Residente Asistencial-Ginecología y Obstetricia; Hospital Carlos Andrade Marín; Quito, Ecuador ${ }^{3}$.
}

\section{RESUMEN}

Objetivos: 1) Determinar la prevalencia de embarazo ectópico en el Servicio de Alto Riesgo Obstétrico del Hospital Carlos Andrade Marín. 2) Identificar los factores de riesgo asociados con esta patología. Métodos: se realizó un estudio descriptivo, de corte transversal, retrospectivo, tomando en cuenta a las pacientes ingresadas con diagnóstico de Embarazo Ectópico al Servicio de Alto Riesgo del Hospital Carlos Andrade Marín, en el periodo del 1 de enero del 2017 al 31 de mayo del 2019. Resultados: durante el período comprendido entre el 1 de enero de 2017 y el 31 de mayo de 2019, se registró un total de 7.596 nacimientos en el Servicio de Alto Riesgo Obstétrico del Hospital Carlos Andrade Marín; de ellos, hubo 117 pacientes diagnosticadas de embarazo ectópico. La prevalencia del embarazo ectópico en el Hospital Carlos Andrade Marín, entre los años 2017 y 2019, fue de 1,5\%; la tasa de embarazo ectópico es de 15,40 / 1.000 gestaciones. Conclusiones: el análisis de los datos obtenidos de las historias clínicas evidenció que la tasa de prevalencia de embarazo ectópico en el Servicio de Alto Riesgo Obstétrico del Hospital Carlos Andrade Marín es similar a la reportada por diferentes estudios realizados alrededor del mundo.

Palabras claves: embarazo ectópico, prevalencia, trompa de falopio, factores de riesgo.

\section{ABSTRACT}

Objective: To determine the prevalence of ectopic pregnancy in the high-risk obstetric service of the Carlos Andrade Marín Hospital and to know the associated risk factors. Methods: A descriptive, cross-sectional, retrospective study was carried out, taking into account patients admitted with a diagnosis of ectopic pregnancy to the high-risk service of the Carlos Andrade Marín Hospital, from January 1, 2017 to May 31 of 2019. Results: During the period from January 1, 2017 to May 31, 2019, a total of 7596 births were recorded in the High Obstetric Risk Service of Hospital Carlos Andrade Marín. Of all of them, a total of 117 patients were registered diagnosis of ectopic pregnancy. The prevalence of ectopic pregnancies in the Hospital Carlos Andrade Marín in a period between 2017 and 2019 was 1.5\%, with an ectopic pregnancy rate of 15.40 per 1000 pregnancies. Conclusions: When performing an analysis of the data obtained from the medical records it was possible to obtain similar results of prevalence rates of ectopic pregnancy in the High Risk Obstetric Service of the Hospital Carlos Andrade Marín in relation to the data obtained by different studies conducted around of the world.

Keywords: ectopic pregnancy, prevalence, fallopian tube, risk factors.

Darwin Logroño: Jorge Ramírez: Adriana Campoverde:

\section{IDs Orcid}

https://orcid.org/0000-0002-9721-4720 https://orcid.org/0000-0002-5895-3811 https://orcid.org/0000-0001-7878-1917

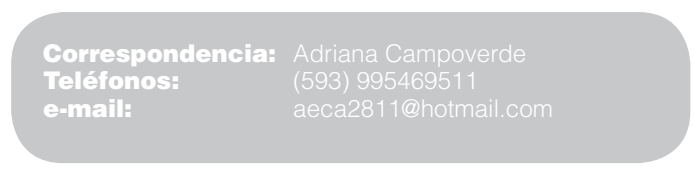




\section{INTRODUCCIÓN}

El embarazo ectópico se produce cuando el blastocisto en desarrollo se implanta a otro nivel distinto del endometrio de la cavidad uterina ${ }^{1}$. La localización extrauterina más frecuente es la trompa de Falopio en 96\% de todos los embarazos ectópicos². El embarazo ectópico es un problema de salud importante de las mujeres en edad fértil. La incidencia varía según la población; se ha contabilizado entre 1 y $2 \%$ de todos los embarazos informados ${ }^{1,3}$.

A pesar de las mejoras de diagnóstico y manejo, la ruptura del embarazo ectópico es aún una causa importante de morbimortalidad relacionada con la gestación. Durante los años 2011 a 2013, la ruptura de embarazos ectópicos se produjo en el 2,7\% de todas las muertes que ocurrieron en el primer trimestre de la gestación $n^{3,4}$.

La incidencia actual es difícil de estimar porque muchas pacientes son tratadas de forma ambulatoria; sin embargo, algunos estudios señalan la incidencia de esta patología. La prevalencia de embarazo ectópico en las mujeres que acuden al servicio de emergencia con metrorragia durante el primer trimestre de gestación, dolor o ambos, oscila entre 6 y $16 \%{ }^{5}$. Un estudio en la red de salud de EE.UU, entre 1997 y 2000, mostró una tasa de 20,7/1.000 gestacopmes. ${ }^{6}$ Otro estudio llevado a cabo en los beneficiarios de seguros de salud, realizado en 14 estados de EE.UU, desde 2004 hasta 2008, informó una tasa de 14,7/1.000 embarazos ${ }^{7}$. Hay muchos factores implicados en la reproducción humana cuya modificación puede repercutir en la tasa de embarazo ectópico; v.gr.: disminución de la natalidad (reduce el denominador de los cálculos de frecuencia que emplean el número de gestaciones), prevalencia en el uso de ciertos métodos anticonceptivos, tratamientos de esterilidad que aumentan el riesgo de embarazo ectópico y mejora de los métodos diagnósticos.

\section{Localización}

Según las Guías NICE 2012, 11/1.000 gestaciones son ectópicas; la localización más frecuente, en 95\% de casos, es la tubárica, particularmente la ampular (80\%). El 5\% restante es extratubárico; las 6 principales localizaciones de las gestaciones no tubáricas son: cervical $(<1 \%)$, intersticial $(2 \%)$, sobre cicatriz de cesárea $(<0,6 \%)$, ovárica $(<1 \%)$ y abdominal $(<1 \%)^{8}$. El embarazo ectópico también puede coexistir con un embarazo intrauterino; esta condición se denomina "embarazo heterotópico" 1 .

\section{Etiopatogenia}

La trompa de Falopio participa en el proceso de reproducción: conduce los espermatozoides al sitio de fecundación y, una vez que ésta ocurre, transporta el óvulo fecundado de retorno hacia la cavidad uterina.
Los procesos que pueden alterar la función tubárica a menudo son inflamatorios, pero pueden ser traumáticos, congénitos y tumorales. La distorsión anatómica puede acompañarse de deterioro de la actividad ciliar de las trompas uterinas. La enfermedad pélvica inflamatoria (EPI) es un factor etiológico importante; la causa traumática engloba la cirugía de trompas y la esterilización quirúrgica.

La incidencia del embarazo ectópico, después de una esterilización quirúrgica, es de 15 a 20\%.

De las causas adquiridas, la endometriosis es una de las más frecuentes; de las tumorales, la salpingitis ístmica nodosa es la más común. Durante años se consideró que la anticoncepción con dispositivo intrauterino (DIU) era un factor de riesgo de embarazo ectópico; sin embargo, en estudios realizados se pudo observar que el riesgo es el mismo en las mujeres que utilizaban DIU que en los controles idénticos, salvo cuando el dispositivo contiene progesterona ${ }^{9}$.

\section{Factores de riesgo}

La mitad de todas las mujeres diagnosticadas de embarazo ectópico no tiene factores de riesgo conocidos $^{10}$.

Aquellas con antecedentes de embarazo ectópico previo tienen mayor riesgo de recurrencia; la posibilidad de recurrencia de un embarazo ectópico en estas mujeres es de aproximadamente $10 \%{ }^{11}$. Las mujeres con antecedentes de EPI tienen un riesgo de embarazo ectópico de aproximadamente 3 veces mayor ${ }^{12,13}$.

Las mujeres con antecedentes de infertilidad también tienen mayor riesgo de embarazo ectópico, independientemente del método utilizado ${ }^{11}$.

Las mujeres que usan un DIU tienen menor riesgo de embarazo ectópico que aquellas que no usan ningún tipo de método anticonceptivo, ya que los dispositivos son muy efectivos para prevenir la gestación; no obstante, hasta $53 \%$ de embarazos que se asocian con un DIU colocado son ectópicos ${ }^{14}$.

Las mujeres que usan anticoncepción hormonal o un dispositivo intrauterino (DIU) tienen un riesgo muy bajo de concebir cualquier embarazo, sea intrauterino o ectópico. Sin embargo, si conciben, la probabilidad de embarazo ectópico es generalmente mayor que en las mujeres que no usan anticonceptivos ${ }^{15,16,17}$.

\section{Manifestaciones clínicas y diagnóstico}

Todas las mujeres sexualmente activas en edad reproductiva que se presentan con dolor abdominal o sangrado vaginal deben ser examinadas en busca de embarazo, independientemente de si están usando anticonceptivos o no. 
El diagnóstico de embarazo ectópico se complica por el gran espectro de cuadros clínicos, desde las pacientes asintomáticas hasta las que experimentan abdomen agudo y choque hemodinámico ${ }^{18}$. La triada sintomática de embarazo ectópico es amenorrea, sangrado vaginal y dolor abdominal en el primer trimestre de la gestación. Aproximadamente en el 10\% los síntomas son muy inespecíficos o ausentes. En la gestación ectópica accidentada suele aparecer distensión abdominal, peritonismo, hemoperitoneo o shock hemorrágico ${ }^{19}$. El dolor abdominal está presente en el 90\% de los casos; se lo describe como dolor de comienzo sordo y de escasa duración, se localiza en la fosa ilíaca homolateral al embarazo ectópico y conforme pasa el tiempo aumenta la duración e intensidad y se generaliza al abdomen inferior. El paso de sangre al peritoneo incrementa el dolor y cambia sus características (se puede irradiar al hombro por irritación diafragmática del nervio frénico) $)^{9,18}$

La amenorrea, que en ocasiones tiene cierta duración, es frecuente ( 75 a 95\% de los casos), pero puede estar ausente, aunque la supuesta regla se la describa como un sangrado atípico debido a su menor duración y cantidad. El otro síntoma cardinal en frecuencia es la hemorragia vaginal irregular (50 a 80\%); típicamente suele ser descrita como un "manchado". Otros síntomas posibles son los propios de una gestación precoz, como náusea, vómito y turgencia mamaria. El estímulo hormonal, procedente del trofoblasto o del cuerpo lúteo, agranda el útero en 20 a $30 \%$ de los casos, aunque el tamaño no se correlaciona con el tiempo de amenorrea $^{9,18}$.

El hemoperitoneo produce irritación peritoneal que se manifiesta por dolor de rebote sin defensa abdominal. El hematocele retrouterino explica el dolor desencadenado por la presión en el fondo de saco vaginal posterior y al movilizar el cérvix desde la vagina ${ }^{9,18}$.

\section{Ecografía transvaginal}

Según diferentes estudios; ${ }^{19}$ la ecografía transvaginal es el método de diagnóstico precoz más sensible de la gestación ectópica (sensibilidad: 87 a 99\%; especificidad: 94 a 99,9\%). El diagnóstico certero de la gestación ectópica se logra mediante la visualización extrauterina de un saco gestacional con vesícula vitelina y/o embrión con o sin latido cardíaco en el $20 \%$ de los casos $^{1,19}$. El hallazgo más común (60\% de los casos) es la imagen heterogénea que se moviliza separadamente del ovario. Generalmente es esférica o elongada y se denomina "blob sign". La sensibilidad y especificidad de estas imágenes diagnósticas de gestación ectópica tubárica es de $89,9 \%$ y $83 \%$ ("blob sign") y de $95,5 \%$ y $99,6 \%$ ("bagel sign"), respectivamente. El Doppler color no contribuye de forma eficaz al diagnóstico de gestación ectópica ${ }^{1,19}$. En más del $20 \%$ de casos se puede visualizar una imagen de "pseudosaco", aunque una imagen similar también puede corresponder a una gestación intrauterina inicial11,19. La presencia de líquido libre hiperecogénico en la pelvis se visualiza en 28 a $56 \%$ de casos de gestación ectópica y corresponde al hemoperitoneo. ${ }^{19}$

\section{Medición sérica de la gonadotropina coriónica humana}

Las mediciones seriadas de hCG se las debe utilizar para diferenciar los embarazos anormales. Cuando los hallazgos sugieren una gestación anormal, se recomienda realizar una segunda medición de hCG, 2 días después de la inicial para evaluar si hay aumento o disminución de la hormona; las evaluaciones subsiguientes de la concentración de hCG deben obtenerse con 2 a 7 días de diferencia ${ }^{1}$. En el embarazo temprano, un aumento de la hCG sérica menor al mínimo del umbral en 48 horas es sospechoso de embarazo anormal (embarazo ectópico o pérdida precoz del embrión) porque el 99\% de los embarazos intrauterinos normales suelen presentar una tasa rápida de aumento. La disminución de los valores de hCG sugiere un embarazo fallido y puede usarse para monitorizar una resolución espontánea, aunque esta disminución no debe considerarse diagnóstica. ${ }^{1}$ Los niveles séricos de hCG $\geq 2.400$ mIU / ml en el 1er trimestre de gestación, sin evidencia de un saco gestacional intrauterino, es diagnóstico de embarazo anormal y muy sugestivo de embarazo ectópico ${ }^{22}$. Las concentraciones séricas de progesterona también pueden ser útiles como complemento de los valores de hCG en la evaluación del embarazo ectópico. El nivel de progesterona $\geq 25 \mathrm{ng}$ / $\mathrm{ml}$ se asocia con un embarazo intrauterino en el 97,5\% de los $\operatorname{casos}^{23}$. Los niveles de progesterona $\leq 5,0 \mathrm{ng} /$ $\mathrm{ml}$ indican embarazo no viable, independientemente de su ubicación ${ }^{22}$

\section{Tratamiento}

Aunque los abordajes quirúrgicos son el tratamiento estándar, los avances en el diagnóstico temprano han facilitado la introducción de la terapia medicamentosa con metotrexato ${ }^{24}$. El uso rutinario de la ecografía temprana ha permitido diagnosticar precozmente el embarazo ectópico y son muchos estos casos en los cuales se puede manejar mediante tratamiento médico. La tasa general de éxito del tratamiento médico en las mujeres adecuadamente seleccionadas es de casi $90 \%{ }^{25}$. El manejo del embarazo ectópico abarca cirugía (salpingostomía o salpingectomía), tratamiento con metotrexato o expectación. Aproximadamente 2/3 de las pacientes con embarazo ectópico requerirán de cirugía y el $1 / 3$ restante serán candidatas para tratamiento con metotrexato ${ }^{6}$. Las candidatas de tratamiento médico con metotrexato deben cumplir las siguientes características: encontrarse hemodinámicamente estables, no tener contraindicaciones para recibir metotrexato, concentración de gonadotropina coriónica 
humana en suero (hCG) $\leq 5.000$ miliUl / $\mathrm{ml}$ y no detectar actividad cardíaca fetal en la ecografía transvaginal. El tamaño de la masa ectópica debe ser menor de 4 $\mathrm{cm}$, no debe haber líquido peritoneal, estar dispuesto y ser capaz de cumplir con el seguimiento posterior al tratamiento, además de tener acceso a los servicios médicos de emergencia dentro de un plazo razonable si hubiere ruptura de la trompa de Falopio. La tasa de éxito cuando se administra una sola dosis, es del $87,2 \%$; logra una permeabilidad tubárica de $81 \%$ de casos tratados ${ }^{1,26,27,28}$. Hay 2 opciones de abordaje quirúrgico para el embarazo tubárico: salpingectomía (extirpación de las trompas de Falopio) y salpingostomía (incisión de la trompa uterina para extraer el embrión dejando la trompa de Falopio intacta y permeable) $)^{29,30}$.

\section{MATERIALES Y MÉTODOS}

El presente estudio descriptivo, de corte transversal y retrospectivo se llevó a cabo en el área de Alto Riego Obstétrico del Hospital Carlos Andrade Marín. Abarcó el período comprendido entre el 1 de enero de 2017 y el 31 de mayo de 2019. Se revisaron las historias clínicas de todas las pacientes que llegaron a esta casa de salud durante este lapso y se tomó en cuenta a todas las pacientes registradas con diagnóstico de embarazo ectópico. El método para calcular la prevalencia de embarazo ectópico en esta casa de salud tomó como denominador el número de nacimientos durante el lapso mencionado. Las variables a identificar fueron: edad, gestas, partos, cesáreas, abortos, embarazo ectópico anterior, edad gestacional, raza, valores de beta-hCG, progesterona, infecciones de transmisión sexual, número de parejas sexuales, inicio de la vida sexual, planificación familiar, dolor pélvico, sangrado vaginal, laparotomía, laparoscopia, ubicación y hemoperitoneo. La información se tabuló en una base de datos electrónica de Microsoft Excel y los datos fueron analizados mediante el programa SPSS.

\section{RESULTADOS}

Durante el período comprendido entre el 1 de enero de 2017 y el 31 de mayo de 2019, se registró un total de 7.596 nacimientos en el Servicio de Alto Riesgo Obstétrico del Hospital Carlos Andrade Marín; de ellos, 117 pacientes fueron diagnosticadas de embarazo ectópico. La prevalencia de embarazo ectópico en el Hospital Carlos Andrade Marín entre los años 2017 y 2019, fue del 1,5\%; siendo la tasa de embarazo ectópico de 15,40 / 1.000 embarazos. La edad promedio de diagnóstico de embarazo ectópico fue de 30,3 años (Figura 1). El 33\% de las pacientes tenía un número de 2 gestas y el 20,9\% tenía ya 3 gestaciones al momento del diagnóstico; del total de pacientes, el 36,75\% mencionó que ya había tenido un aborto anterior y el 9,4\% ya había tenido un embarazo ectópico anterior. Al momento del diagnóstico de embarazo ectópico, el 38,9\% de las pacientes aseguraban tener una amenorrea de 6 a 7 semanas (Figura 2).

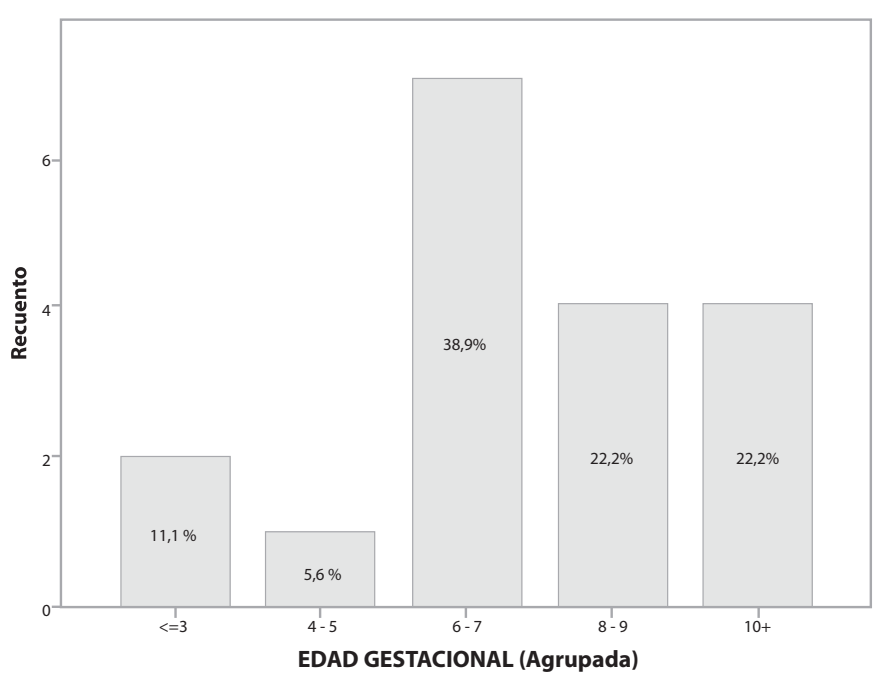

Figura 1. Edad promedio de embarazo ectópico.

Fuente: Los autores

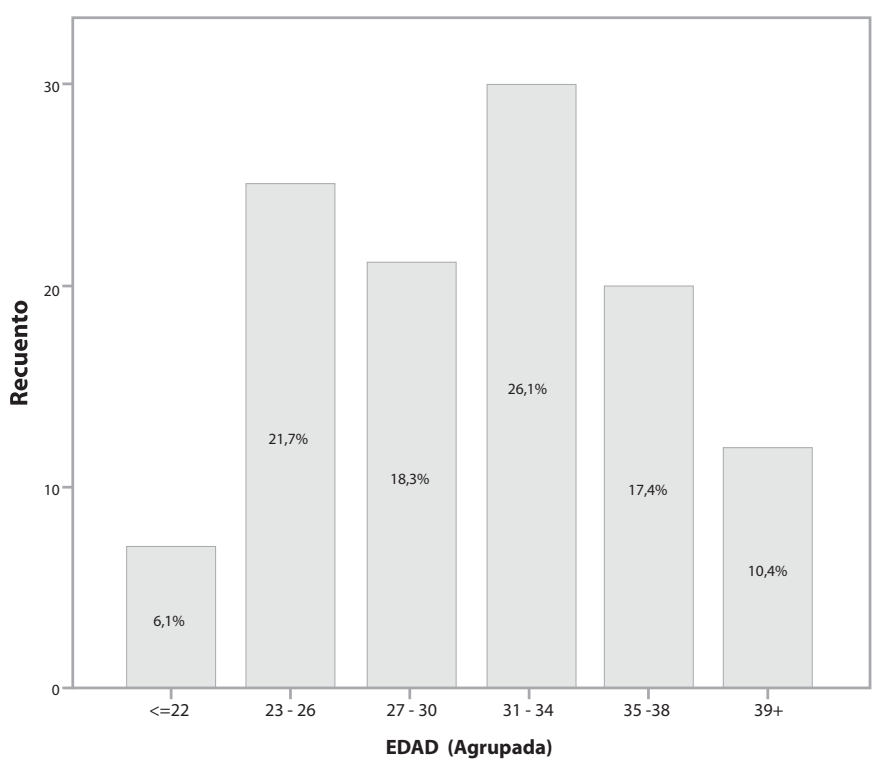

Figura 2. Edad gestacional de embarazo ectópico.

Fuente: Los autores

Al cuantificar los valores de B-hCG, los rangos fueron de 14 a 45.390 (la media de valores de B-hCG al momento del diagnóstico es de $6.220 \mathrm{mlU} / \mathrm{ml}$ ); la mediana es de $1.844 \mathrm{mlU} / \mathrm{ml}$ (Figura 3). El rango de progesterona varía entre 0,2 y 22,4 y su media es de $5,34 \mathrm{ng} / \mathrm{ml}$ y la mediana de 4,27 ng/ml (Figura 4). 


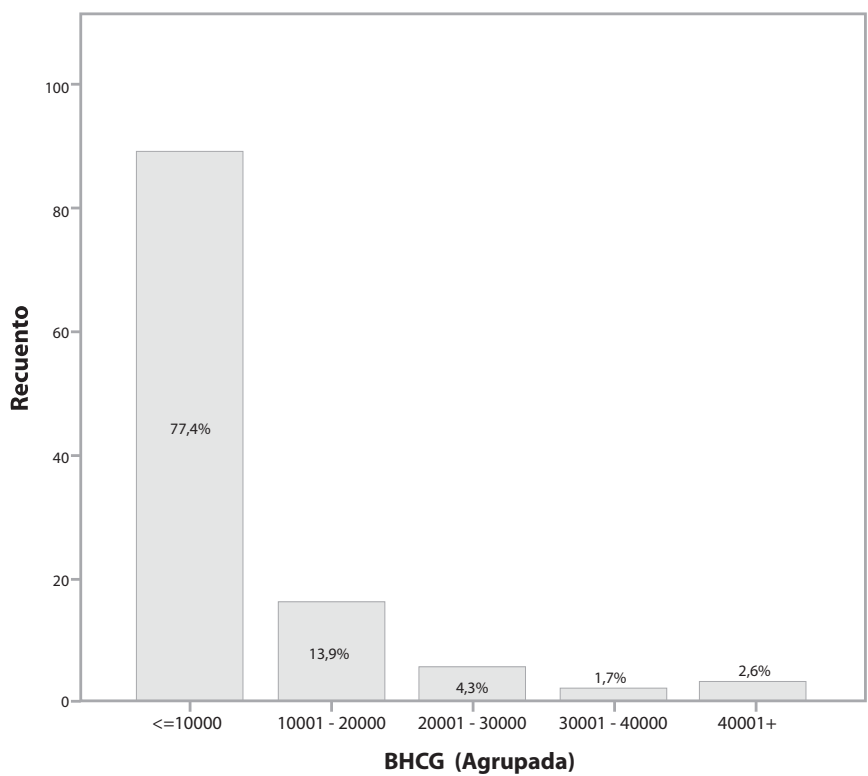

Figura 3. Valores de hormona coriónica humana (B-hcg)

Fuente: Los autores

Sólo el 7\% de las pacientes admitió haber tenido un diagnóstico previo de infección de transmisión sexual; la media del número de parejas sexuales de este grupo fue de 2,92 con una mediana de 2 parejas sexuales; el $46,9 \%$ de las pacientes aseguró haber iniciado su vida sexual entre los 16 y los 18 años (Figura 5). Al momento del diagnóstico de embarazo ectópico, el 28,6\% de las pacientes aseguró encontrarse utilizando un método de planificación familiar (Figura 6).

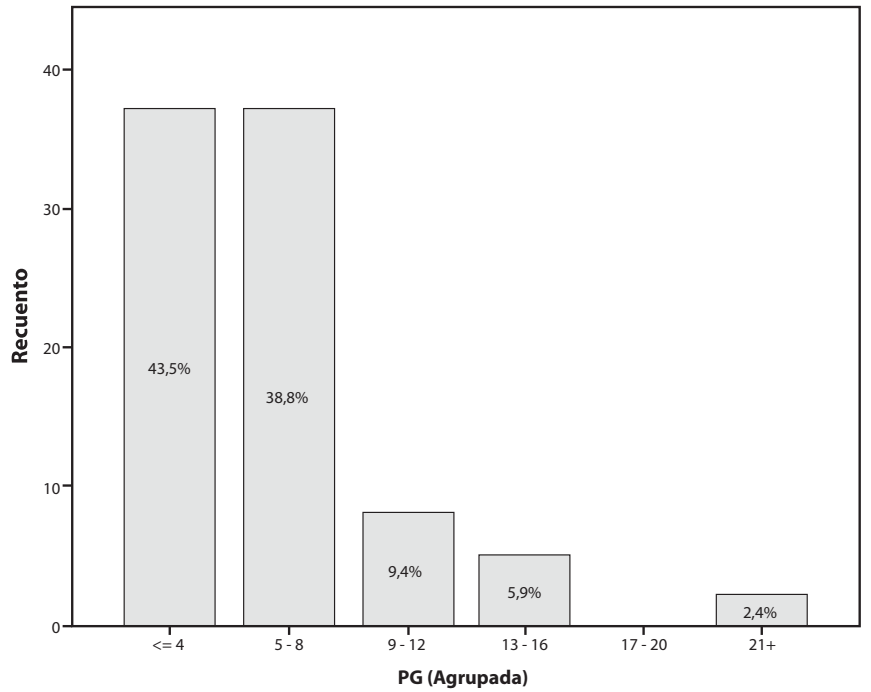

Figura 4. Valores de progesterona (Pg)

Fuente: Los autores

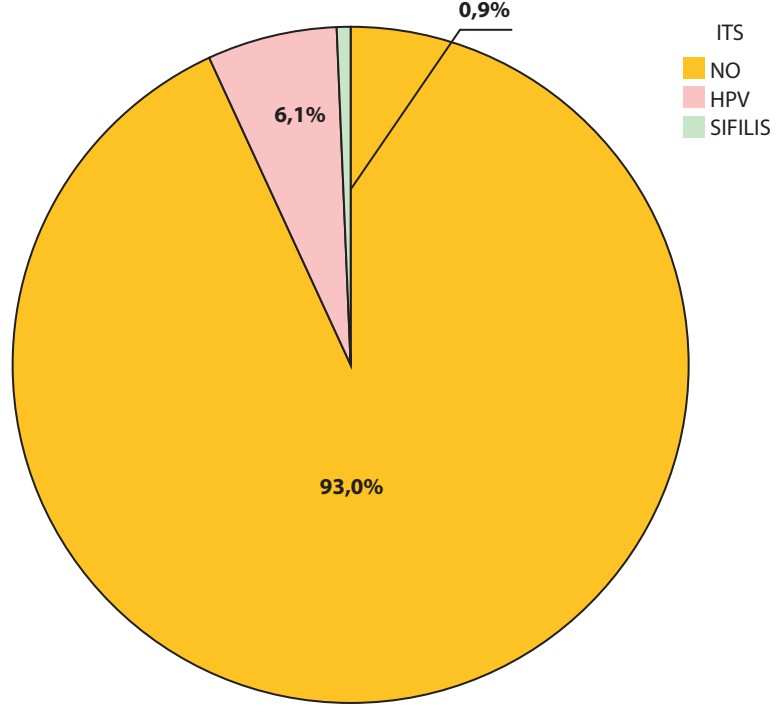

Figura 5. Infecciones de transmisión sexual (ITS) en embarazo ectópico.

Fuente: Los autores

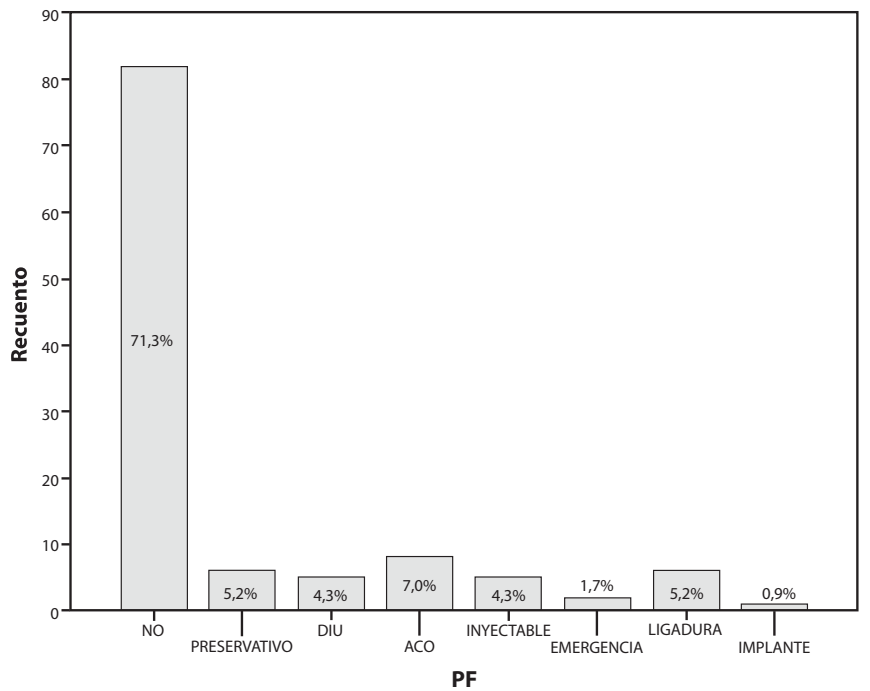

Figura 6. Métodos de planificación familiar.

Fuente: Los autores

Las manifestaciones sintomáticas más frecuentes fueron: dolor pélvico $(95,6 \%$ de las pacientes) y sangrado vaginal (63,7\%). En nuestro registro, la ubicación del embarazo ectópico fue de 99,1\% en las trompas de Falopio y tan sólo el 0,9\% mencionó una ubicación diferente.

\section{Discusıón}

La prevalencia informada de embarazo ectópico ha variado a lo largo del tiempo y en la actualidad es difícil de estimarla porque un porcentaje importante de pacientes recibe tratamiento médico que no requiere de ingreso hospitalario; por este motivo, su verdadera prevalencia se puede subestimar. En el estudio llevado 
a cabo en el Hospital Carlos Andrade Marín se estimó una prevalencia de embarazo ectópico del 1,5\% y una tasa de embarazo ectópico de 15,40/1.000 embarazos; estos datos concuerdan con varios estudios llevados a cabo en el resto del mundo, en los cuales la tasa de embarazo ectópico estimada es de 14,7/1.000 embarazos ${ }^{7}$. El Centro para Enfermedad, Control y Prevención de los Estados Unidos de América (CDC) estima que el embarazo ectópico ocurre en el $2 \%$ de todas las gestacones ${ }^{31}$. Otros estudios mencionan que las tasas diagnósticas pueden ser mayores, posiblemente de 20,7/1.000 embarazos; inclusive hay registros que pueden oscilar entre 6 y 16\%5,6.

La mitad de todas las mujeres diagnosticadas de embarazo ectópico no tiene factores de riesgo conocidos. En nuestro estudio se puede evidenciar que el $9,4 \%$ de las pacientes tuvo ya un embarazo ectópico anterior; éste es un factor importante de tomar en cuenta ya que, según la literatura médica, las mujeres con antecedente de embarazo ectópico tienen mayor riesgo de recurrencia; la probabilidad de repetición es de aproximadamente $10 \%{ }^{1}$. Es importante observar que el $54,8 \%$ de los embarazos ectópicos en nuestro estudio ocurren en mayores de 30 años de edad. Este resultado concuerda con algunos estudios previos que encontraron que el riesgo aumenta con la edad materna avanzada. La evidencia de cómo la edad materna avanzada se relaciona con el riesgo de embarazo ectópico aún nos es clara. Algunos investigadores lo atribuyen a factores relacionados con la edad, posible cicatrización de las trompas por EPI, infecciones por gonococos y clamidias y cambios de la función ciliar de las trompas; no obstante, estas hipótesis deben ser investigadas $32,33,34,35$.

En el presente estudio, el $36,75 \%$ de las pacientes aseguró haber tenido un aborto previo, siendo este un valor representativo; aunque la literatura médica no lo considera como factor de riesgo, podría haber una causa relacionada con una alteración hormonal o factores inmunitarios ${ }^{36,37}$. Es importante destacar que el $28,6 \%$ de las pacientes de nuestro estudio, desarrolló embarazo ectópico asegurando encontrarse usando un método anticonceptivo; el $7 \%$ de éstas aseguró que usaba anticonceptivos orales combinados y el $1,7 \%$ anticoncepción de emergencia. Aunque son muy efectivos y el riesgo general de embarazo ectópico es bajo, ya que evita la concepción, en las mujeres que quedan embarazadas mientras toman estos anticonceptivos el riesgo de embarazo ectópico parece aumentar de 2 a 5 veces en comparación con otras mujeres gestantes; más aún, con los anticonceptivos orales de progestágeno, un estudio encontró un riesgo de 4 a 79 embarazos ectópicos /1.000 mujeres / año ${ }^{16,17}$. En nuestro estudio, el 5,2\% de pacientes con embarazo ectópico se realizó esterilización quirúrgica previa; según la literatura médica, la esterilización laparoscópica con uso de bipolar tuvo un riesgo informado de embarazo ectópico de 8,4/1.000 procedimientos, tanto a los 5 años como a los 10 años de la intervención quirúrgica. ${ }^{38,39}$ El 4,3\% de pacientes mencionaron utilizar dispositivos intrauterinos, lo que concuerda con la literatura médica; sin embargo, aunque son muy efectivos y el riesgo general de embarazo ectópico es bajo (pues evita la concepción) entre las usuarias de DIU con insuficiencia anticonceptiva, el riesgo de embarazo ectópico es alto ( 1 de 2 gestaciones para el DIU de levonorgestrel y 1 de 16 para el DIU de cobre). ${ }^{40}$

Es importante precisar que la triada diagnostica clásica de embarazo ectópico es dolor abdominal (en nuestro estudio lo refirieron el 95,6\% de pacientes), sangrado vaginal $63,7 \%$ amenorrea $77,7 \%$; según la literatura médica, estos signos clásicos suelen ser constantes. El dolor abdominal es el síntoma clásico (90\% de casos), seguido de la amenorrea (75 a 95\%) y hemorragia vaginal $(50 \text { a } 80 \%)^{9}$. Es importante mencionar que el estudio complementario con exámenes de laboratorio puede orientar nuestro diagnóstico de embarazo ectópico. En nuestro estudio, se observa una mediana del valor de B-hCG de $1.844 \mathrm{mlUl} / \mathrm{ml}$ y de progesterona de $4,27 \mathrm{ng} / \mathrm{ml}$; los valores de B-hCG, demuestran un rango muy amplio por lo cual no se puede asegurar que un determinado nivel de referencia nos ayudará a sospechar el diagnóstico. Se deberían correlacionar estos valores con los hallazgos ecográficos llevados a cabo vía transvaginal o correlacionarlos con tomas seriadas de B-hCG para confirmar o descartar el diagnóstico en concordancia con la recomendación de la literatura actual. Los valores de progesterona en nuestras pacientes son homogéneos; la mediana de su concentración es de $4,27 \mathrm{ng} / \mathrm{ml}$, que nos permite afirmar que las concentraciones de progesterona menores de $5,0 \mathrm{ng} / \mathrm{ml}$ sugieren que el embarazo es no viable ${ }^{22}$.

\section{CONCLUSIONES}

La prevalencia estimada de embarazo ectópico en el Servicio de Alto Riesgo del Hospital Carlos Andrade Marín en el período comprendido entre el 1 de enero de 2017 y el 31 de mayo de 2019 es de 1,5, y la tasa es de $15,40 / 1.000$ gestaciones.

Los factores de riesgo asociados con el embarazo ectópico deben ser investigados en la anamnesis con el fin de lograr un diagnóstico preciso.

En una paciente gestante que, durante el primer trimestre, presente la triada clásica de dolor abdominal, hemorragia vaginal y amenorrea se debe tener alta sospecha de la posibilidad de embarazo ectópico.

Los valores séricos de B-hCG por sí solos no deben utilizarse como recurso diagnóstico de embarazo ectópico y es preciso correlacionarlos con la historia clínica y la ecografía. 
El embarazo ectópico es una patología frecuentemente responsable de la morbimortalidad materna; en consecuencia, el diagnóstico temprano permitirá tomar decisiones oportunas.

\section{CONTRIBUCIÓN DE LOS AUTORES}

Logroño Darwin: Concepción y diseño del trabajo; recolección y obtención de resultados; análisis e interpretación de datos; redacción del manuscrito.

Ramírez Jorge: Concepción y diseño del trabajo; recolección y obtención de resultados; análisis e interpretación de datos; redacción del manuscrito.

Campoverde Adriana: Concepción y diseño del trabajo; recolección y obtención de resultados; análisis e interpretación de datos; redacción del manuscrito.

\section{CONFLICTO dE INTERÉS}

Los autores declararon no tener ningún conflicto de interés personal, financiero, intelectual, económico y de interés corporativo con el Hospital Metropolitano y los miembros de la revista MetroCiencia.

\section{REFERENCIAS BIBLIOGRÁFICAS}

1. Barnhart KT, Franasiak JM. Tubal ectopic pregnancy. ACOG Practice Bulletin No. 191. American College of Obstetricians and Gynecologists. Obstet Gynecol 2018;131:e65-77. https://doi.org/10.1097/ aog.0000000000002464

2. Bouyer J, Coste J, Fernandez H, Pouly JL, Job-Spira N. Sites of ectopic pregnancy: a 10 years population-based study of 1800 cases. Hum Reprod 2002;17(12):3224-3230. https://doi.org/10.1093/humrep/17.12.3224

3. Beera Neelima, VG Vanamala. Risk factors of ectopic pregnancy: a study in a tertiary care centre. 2017.

4. Creanga AA, Syverson C, Seed K, Callaghan WM. Pregnancy-related mortality in the United States, 2011-2013. Obstet Gynecol 2017;130:366 373. https://doi.org/10.1097/aog.0000000000002114

5. Cabrera Samith I, Perales I, Romero G. Análisis epidemiológico y clínico del embarazo ectópico: Hospital Base de Los Ángeles. Rev Chil Obstet Ginecol. [Internet]. 2010 [citado 2019 Jul 04]; 75(2): 96-100. http:// dx.doi.org/10.4067/S0717-75262010000200004

6. Van Den Eeden SK, Shan J, Bruce C, Glasser M. Ectopic pregnancy rate and treatment utilization in a large managed care organization. Obstet Gynecol 2005;105:1052. https://doi.org/10.1097/01. aog.0000158860.26939.2d

7. Stulberg DB, Cain LR, Dahlquist I, Lauderdale DS. Ectopic pregnancy rates and racial disparities in the Medicaid population, 2004-2008. Fertil Steril 2014;102:1671. https://dx.doi.org/10.1016\%2Fj.fertnstert.2014.08.031

8. Parker VL, et al. Non-tubal ectopic pregnancy. Arch GynecolObstet 2016;294:19-27. https://doi.org/10.1007/s00404-016-4069-y

9. Usandizaga J, De la Fuente. Obstetricia y Ginecología. 2da. edición. España. Marbán. 2011;pp:310-320.

10. Barnhart KT, Sammel MD, Gracia CR, Chittams J,Hummel AC, Shaunik A. Risk factors for ectopic pregnancy in women with symptomatic first-trimester pregnancies. Fertil Steril 2006;86:36-43. https://doi.org/10.1016/i. fertnstert.2005.12.023

11. Ankum WM, Mol BW, Van der Veen F, Bossuyt PM. Risk factors for ectopic pregnancy: a meta-analysis. Fertil Steril 1996;65:1093-1099. Disponible: https://pubmed.ncbi.nlm.nih.gov/8641479/

12. Kamwendo F, Forslin L, Bodin L, Danielsson D. Epidemiology of ectopic pregnancy during a 28 years period and the role of pelvic inflammatory disease. Sex Transm Infect 2000;76:28. https://doi.org/10.1136/ sti.76.1.28
13. Davies B, Turner KM, Frølund $\mathbf{M}$, et al. Risk of reproductive complications following chlamydia testing: a population-based retrospective cohort study in Denmark. Lancet Infect Dis 2016;16:1057. https://doi. org/10.1016/s1473-3099(16)30092-5

14. Backman T, Rauramo I, Huhtala S, Koskenvuo M. Pregnancy during the use of levonorgestrel intrauterine system. Am J Obstet Gynecol 2004; 190:50-54. https://doi.org/10.1016/j.ajog.2003.07.021

15. Sivin I. Dose- and age-dependent ectopic pregnancy risks with intrauterine contraception. Obstet Gynecol 1991;78:291. Disponible en: https:// pubmed.ncbi.nlm.nih.gov/2067778/

16. Li C, Zhao WH, Meng CX, et al. Contraceptive use and the risk of ectopic pregnancy: A multi-center case-control study. PLoS One 2014;9:e115031. https://doi.org/10.1371/journal.pone.0115031

17. Larimore WL, Stanford JB. Postfertilization effects of oral contraceptives and their relationship to informed consent. Arch Fam Med 2000;9:126. https://doi.org/10.1001/archfami.9.2.126

18. Berek, Jonathan S. Berek y Novak Ginecología. 15 va edición. España. Lippincott Castellano. 2013;pp.421.

19. Protocolos Medicina Maternofetal. Hospital Clínic-Hospital Sant Joan de Déu- Universitat de Barcelona. Protocolo: Gestación ectópica tubárica y no tubárica. 2018. Disponible en: www.medicinafetalbarcelona.org

20. Barnhart K, Sammel MD, Chung K, Zhou L, Hummel AC, Guo W. Decline of serum human chorionic gonadotropin and spontaneous complete abortion: defining the normal curve. Obstet Gynecol 2004;104:975-981. https://doi.org/10.1097/01.aog.0000142712.80407.fd

21. Kadar N, Romero R. Serial human chorionic gonadotropin measurements in ectopic pregnancy. Am J Obstet Gynecol 1988;158:1239-1240. https://doi.org/10.1016/0002-9378(88)90263-3

22. The Practice Committee of the American Society for Reproductive Medicine. Early diagnosis and management of ectopic pregnancy. Feltil Steril 2004 Sep;82(Suppl 1):S146-814.

23. Stovall TG, Ling FW, Cope BJ, Buster JE. Preventing ruptured ectopic pregnancy with a single serum progesterone. Am J Obstet Gynecol 1989;160:1425-1428. https://doi.org/10.1016/0002-9378(89)90866-1

24. Lipscomb GH. Medical therapy for ectopic pregnancy. Semin Reprod Med 2007;25:93-98. https://doi.org/10.1055/s-2007-970048

25. Barnhart KT, Gosman G, Ashby R, Sammel M. The medical management of ectopic pregnancy: a meta-analysis comparing "single dose" and "multidose" regimens. Obstet Gynecol 2003;101:778. https://doi. org/10.1016/s0029-7844(02)03158-7

26. Starndell A, Thorburn J, Hamberger L. Risk factors for ectopic pregnancy in assisted reproduction. Fertl Steril 1999;71:282-286. https://doi. org/10.1016/s0015-0282(98)00441-5

27. Farquhar CM. Ectopic pregnancy. Lancet. 2005;366(9485):583-591. https://doi.org/10.1016/s0140-6736(05)67103-6

28. Hajenius PJ, Mol F, Mol BW, Bossuyt PM, Ankum WM, van der Veen F. Interventions for tubal ectopic pregnancy. Cochrane Database Syst Rev. 2007;2007(1):CD000324. https://doi.org/10.1002/14651858.cd000324. pub2

29. Kirk E, Condous G, Bourne T. The non-surgical management of ectopic pregnancy. Ultrasound Obstet Gynecol 2006;27:91-100. https://doi. org/10.1002/uog.2602

30. Walker J. Guidelines on diagnosing ectopic pregnancy. 2002. The ectopic pregnancytrust. Disponible en: www.ectopic.org

31. Ectopic pregnancy--United States, 1990-1992. Centers for Disease Control and Prevention (CDC). MMWR Morb Mortal Wkly Rep. 1995;44: 46-48.

32. Coste J, Job-Spira N, Fernandez H, Papiernik E, Spira A. Risk factors for ectopic pregnancy: A case-control study in France, with special focus on infectious factors. Am J Epidemiol 1991;133:839-849

33. Mäkinen JI, Erkkola RU, Laippala PJ. Causes of the increase in the incidence of ectopic pregnancy. A study on 1017 patients from 1966 to 1985 in Turku, Finland. Am J Obstet Gynecol 1989;160:642-646.

34. Egger M, Low N, Smith GD, Lindblom B, Herrmann B. Screening for chlamydial infections and the risk of ectopic pregnancy in a county in Sweden: Ecological analysis. BMJ 1998; 316:1776-1780.

35. Kamwendo F, Forslin L, Bodin L, Danielsson D. Epidemiology of ectopic pregnancy during a 28 years period and the role of pelvic inflammatory disease. Sex Transm Infect 2000;76: 28-32. https://doi.org/10.1136/ $\underline{\text { sti.76.1.28 }}$ 
36. Bouyer J, Rachou E, Germain E, Fernandez H, Coste J, Pouly JL, et al. Risk factors for extrauterine pregnancy in women using an intrauterine device. Fertil Steril 2000;74:899-908.

37. Fernandez H, Bouyer J, Coste J, Job-Spira N. The hidden side of ectopic pregnancy: The hormonal factor. Hum Reprod 1996;11:243-234. https://doi.org/10.1093/humrep/11.2.243

38. Malacova E, Kemp A, Hart R, et al. Long-term risk of ectopic pregnancy varies by method of tubal sterilization: a whole-population study. Fertil

Steril 2014;101:728. https://doi.org/10.1016/.fertnstert.2013.11.127

39.

Perkins RB, Morgan JR, Awosogba TP, et al. Gynecologic outcomes after hysteroscopic and laparoscopic sterilization Procedures. Obstet Gynecol 2016;128:843. https://doi.org/10.1097/aog.0000000000001615

40. Li C, Zhao WH, Zhu Q, et al. Risk factors for ectopic pregnancy: a multi-center case-control study. BMC Pregnancy Childbirth 2015;15:187. https://doi.org/10.1186/s12884-015-0613-1

\section{CITAR ESTE ARTICULO}

Logroño D, Ramírez J, Campoverde A. Prevalencia de embarazo ectópico en el Hospital Carlos Andrade Marín, 2017 a 2019. MetroCiencia. 2020 ene; 28(1): p. 58-65. DOI: https://doi. org/10.47464/MetroCiencia/vol28/1/2020/58-65. Available from: https://revistametrociencia.com. ec/index.php/revista/article/view/58 